\title{
Mapping Radius of Regular Function and Center of Convex Region
}

\author{
Duan Wenxi \\ School of Applied Mathematics \\ Beijing Normal University, Zhuhai China \\ 236304613@qq.com
}

\begin{abstract}
Keywords: regular function; mapping radius; conformal transformation; center of convex region
\end{abstract}
\begin{abstract}
There is only a univalent and regular function in a simply connected region. It transforms the region into a unit circle, while satisfies that the function value of a point is equal to zero and the derivative of this point is greater than zero, then the inverse of the derivative is named as the mapping radius of the function at the point. The mapping radius is changing according to the movement of the point. In fact, the mapping radius in the region is a real valued function. The function is continuous and reaches the maximum value within the region. If a simply connected region is the convex region with the symmetrical center, then the mapping radius function of the region obtains the maximum at the symmetrical center of the region.
\end{abstract}

\section{Introduction}

First of all, we look back on the famous Riemann' Theorem and his deduction[1].

Riemann' Theorem: Supposing the boundary points of the simply connected region $G$ on the plane $Z$ are not limited to one, $z_{0} \in G$, there is the only univalent and regular function $f(z)$ that $G$ will be transformed into the unit circle: $|w|<1$ on the plane $W$, and meets

$$
f\left(z_{0}\right)=0, f^{\prime}\left(z_{0}\right)>0 \text {. }
$$

Deduction: Supposing the boundary points of the simply connected region $G$ on the plane $Z$ are not limited to one, $z_{0} \in G$, there is the only univalent and regular function $F(z)$ that $G$ will be transformed to the unit circle:

$$
|w|<\frac{1}{f^{\prime}\left(z_{0}\right)}
$$

on the plane $W$, and meets $F\left(z_{0}\right)=0, F^{\prime}\left(z_{0}\right)=1$.

In fact, suppose $w=f(z)$ is the univalent and regular function with the terms of Riemann' Theorem $F(z)=\frac{f(z)}{f^{\prime}\left(z_{0}\right)}, F\left(z_{0}\right)=0, F^{\prime}\left(z_{0}\right)=1, G$ will be transformed to the circle by the $F(z)$, and

$$
|w|=|f(z)|=\left|\frac{f(z)}{f^{\prime}\left(z_{0}\right)}\right|<\frac{1}{f^{\prime}\left(z_{0}\right)} .
$$

\section{Mapping radius}

Definition: Suppose the function $f(z)$ meets the terms of Riemann’ Theorem, and

$$
R=\frac{1}{f^{\prime}\left(z_{0}\right)}
$$

is named as the mapping radius of $f(z)$ at the point $z_{0}$ of $G$.

We can see from the above deductions, if $G$ will be transformed into the circle: $|w|<R$ by the $F(z)$

( $F\left(z_{0}\right)=0, F^{\prime}\left(z_{0}\right)=1$ ), then the mapping radius at the point $z_{0}$ of $G$ is the radius of the circle: $|w|<R$.

Suppose $G$ is the simply connected region that the boundary points are not limited to one on the plane $Z$, and $z_{0} \in G$. Suppose $N(G)$ is the family of functions which meet the following conditions.

(1) $F(z)$ is the univalent and regular function in the $G$. 
(2) $F\left(z_{0}\right)=0, F^{\prime}\left(z_{0}\right)=1$.

The properties of the family of functions are following:

Property 1 Suppose $M(F)=\sup _{z \in G}|F(z)|$, then there is a univalent and regular function $F(z)$ in $N(G)$ reaches the minimum of $M(F)$. And the minimum value is equal to the mapping radius at the point $z_{0}$ of $G$.

Proof: We are limited to $M(F)<\infty$. Suppose $w=f(z)$ is the univalent and regular function that $G$ will only be transformed to the unit circle: $|w|<1$, and meets $f\left(z_{0}\right)=0, f^{\prime}\left(z_{0}\right)>0$. Its inverse function is marked as $z=\varphi(w)$. Again mark $\psi(w)=\frac{F(\varphi(w))}{M(F)}$, then $\psi(w)$ is the univalent and regular function on the unit circle: $|w|<1, \psi(0)=0,|\psi(w)|<1$, according to Schwarz’ Theorem, $\left|\psi^{\prime}(0)\right| \leq 1$.

because

$$
\psi^{\prime}(w)=\frac{F^{\prime}(\varphi(w))}{M(F)} \varphi^{\prime}(w)=\frac{F^{\prime}(\varphi(w))}{M(F)} \frac{1}{f^{\prime}(z)}, \psi^{\prime}(0)=\frac{F^{\prime}(\varphi(0))}{M(F)} \frac{1}{f^{\prime}\left(z_{0}\right)}=\frac{1}{M(F) f^{\prime}\left(z_{0}\right)},
$$

so $\quad M(F) \geq \frac{1}{f^{\prime}\left(z_{0}\right)}=R$. when $\psi(w)=w,\left|\psi^{\prime}(0)\right|=1, M(F)=\frac{1}{f^{\prime}\left(z_{0}\right)}$, therefore $w=\frac{F(\varphi(w))}{M(F)}$ that is to say, $w=f^{\prime}\left(z_{0}\right) F(z)$, or $f(z)=f^{\prime}\left(z_{0}\right) F(z)$, thus

$$
F(z)=\frac{f(z)}{f^{\prime}\left(z_{0}\right)} .
$$

Property2: When the area $S(F)=\iint_{G}\left|F^{\prime}(z)\right|^{2} d \sigma$ reaches minimum, $M(F)=\sup _{z \in G}|F(z)|$ reaches minimum, and only when $F(z)=\frac{f(z)}{f^{\prime}\left(z_{0}\right)}, S(F)$ is the minimum. Among which $w=f(z)$ is the univalent and regular function that $G$ will transformed to the unit circle: $|w|<1$, and meets $f\left(z_{0}\right)=0, f^{\prime}\left(z_{0}\right)>0$.

Proof : we are limited to $S(F)$ is a finite number ,the inverse function of $w=f(z)$ is $z=\varphi(w)$, $w=r e^{i \theta}$, then

$$
S(F)=\iint_{G}\left|F^{\prime}(z)\right|^{2} d \sigma=\iint_{|w|<1}\left|F^{\prime}(\varphi(w)) \varphi^{\prime}(w)\right|^{2} r d r d \theta=\lim _{\rho \rightarrow 1} \iint_{\mid w<\rho}\left|F^{\prime}(\varphi(w)) \varphi^{\prime}(w)\right|^{2} r d r d \theta
$$

We will $F^{\prime}(\varphi(w)) \varphi^{\prime}(w)$ as power series $F^{\prime}(\varphi(w)) \varphi^{\prime}(w)=\sum_{n=0}^{+\infty} a_{n} w^{n}$,Among which

$$
a_{0}=F^{\prime}(\varphi(0)) \varphi^{\prime}(0)=F^{\prime}\left(z_{0}\right) \frac{1}{f^{\prime}\left(z_{0}\right)}=\frac{1}{f^{\prime}\left(z_{0}\right)}
$$

Pay attention to $\int_{0}^{2 \pi} e^{i(n-m) \theta} d \theta=\left\{\begin{array}{cc}0 & m \neq n \\ 2 \pi & m=n\end{array}\right.$

$$
\begin{array}{lr}
\iint_{\mid w<\rho}\left|F^{\prime}(\varphi(w)) \varphi^{\prime}(w)\right|^{2} r d r d \theta=\iint \sum_{|w|<\rho} \sum_{n=0}^{+\infty} a_{n} w^{n} \cdot \sum_{m=0}^{+\infty} \overline{a_{n} w^{m}} r d r d \theta=\iint_{|w|<\rho} \sum_{n, m=0}^{+\infty} a_{n} \overline{a_{m}} r^{n+m+1} e^{i(n-m) \theta} d \theta d r \\
=\sum_{n, m=0}^{+\infty} \int_{0}^{2 \pi} e^{i(n-m) \theta} d \theta \int_{0}^{\rho} a_{n} \overline{a_{m}} r^{m+n+1} d r & =\sum_{n=0}^{+\infty} 2 \pi\left|a_{n}\right|^{2} \int_{0}^{\rho} r^{2 n+1} d r=\sum_{n=0}^{+\infty} \pi\left|a_{n}\right|^{2} \frac{\rho^{2 n+2}}{n+1} \\
S(F)=\lim _{\rho \rightarrow 1} \iint_{|w|<\rho} \mid F^{\prime}\left(\left.\varphi(w) \varphi^{\prime}(w)\right|^{2} r d r d \theta\right. & \\
=\lim _{\rho \rightarrow 1} \sum_{n=0}^{+\infty} \pi\left|a_{n}\right|^{2} \frac{\rho^{2 n+2}}{n+1}=\sum_{n=0}^{+\infty} \frac{\pi\left|a_{n}\right|^{2}}{n+1} \geq \pi\left|a_{0}\right|^{2}
\end{array}
$$

When $a_{1}=a_{2}=\cdots=0$, the $S(F)=\pi\left|a_{0}\right|^{2}$, in this case , $F^{\prime}[\varphi(w)] \varphi^{\prime}(w)=a_{0}, \quad F(\varphi(w))=a_{0} w$, $F(z)=a_{0} f(z)$, so

$$
F(z)=f(z) / f^{\prime}\left(z_{0}\right) .
$$




\section{Mapping radius functions of the circle}

Suppose $G:|z-a|<R$ is the circle on the plane $Z, z_{0} \in G$. The function

$$
f(z)=R \frac{z-z_{0}}{R^{2}-\left(\overline{z_{0}}-\bar{a}\right)(z-a)}
$$

will transformed the circle $G$ into the unit circle: $|w|<1$ on the plane $W$, and meets

$$
f\left(z_{0}\right)=0, f^{\prime}\left(z_{0}\right)=\frac{R}{R^{2}-\left|z_{0}-a\right|^{2}}>0 .
$$

The mapping radius at point $z_{0}$ of $G:|z-a|<R_{0}$ is

according to the definition.

$$
\frac{1}{f^{\prime}\left(z_{0}\right)}=\frac{R^{2}-\left|z_{0}-a\right|^{2}}{R}
$$

If $z_{0}$ is looked upon as the moving point, the mapping radius of the circle $G:|z-a|<R$ is

$$
R(z)=\frac{R^{2}-|z-a|^{2}}{R}
$$

Obviously, the mapping radius function of the circle has the following properties:

(1) $R(z)$ is the monotone decreasing function of $|z-a|=r$;

(2) $R(z)$ obtains the maximum at the center of the circle, and $\max _{z \in G} R(z)=R_{0}$.

\section{Mapping radius function of the simply connected region}

Suppose $G$ is the simply connected region on the plane $Z$ which the boundary points are not limited to one. $z_{0} \in G$, the univalent and regular function $f(z)$ in the $G$ will make $G$ transformed into the unit circle: $|w|<1$ on the plane $w$, and meet $f\left(z_{0}\right)=0, f^{\prime}\left(z_{0}\right)>0$. For $a \in G$, the following function is made:

$$
\begin{aligned}
g(z) & =e^{i \theta} \frac{f(z)-f(a)}{1-f(z) \overline{f(a)}}, \\
g^{\prime}(z) & =e^{i \theta} \frac{f^{\prime}(z)[1-f(z) \overline{f(a)}]+f^{\prime}(z)[f(z)-f(a)] \overline{f(a)}}{[1-f(z) \overline{f(a)}]^{2}}=e^{i \theta} \frac{f^{\prime}(z)[1-f(z) \overline{f(a)}+f(z) \overline{f(a)}-f(a) \overline{f(a)}]}{[1-f(z) \overline{f(a)}]^{2}} \\
& =e^{i \theta} \frac{f^{\prime}(z)[1-f(a) \overline{f(a)}]}{[1-f(z) \overline{f(a)}]^{2}} . \\
g^{\prime}(a) & =e^{i \theta} \frac{f^{\prime}(a)[1-f(a) \overline{f(a)}]}{[1-f(a) \overline{f(a)}]^{2}}=e^{i \theta} \frac{f^{\prime}(a)}{1-f(a) \overline{f(a)}}=e^{i \theta} \frac{f^{\prime}(a)}{1-|f(a)|^{2}},
\end{aligned}
$$

If at first choose $\theta=-\arg f^{\prime}(a)$, thus

$$
g^{\prime}(a)=\frac{\left|f^{\prime}(a)\right|}{1-|f(a)|^{2}}>0 \text {. }
$$

$G$ will also transformed into the unit circle: $|w|<1$ on the plane $W$ by $g(z)$, and $g(a)=0, g^{\prime}(a)>0$. Therefore, the mapping radius of the point $a$ about region $G$ is

$$
\frac{1}{g^{\prime}(a)}=\frac{1-|f(a)|^{2}}{\left|f^{\prime}(a)\right|} \text {. }
$$

The point $a$ is considered as the moving point $z$, thus the mapping radius function in $G$ is obtained as

$$
R_{G}(z)=\frac{1-|f(z)|^{2}}{\left|f^{\prime}(z)\right|}
$$

Since $f^{\prime}(z) \neq 0$, clearly $R_{G}(z)$ is the continuous function in $G$. 


\section{The maximum of the mapping radius function and the center of the convex region}

For $M(F)$ that is introduced in the first paragraph, the properties are following:

Property 1: If $G_{1} \subset G$, thus

$$
\inf _{F \in G_{1}} M(F) \leq \inf _{F \in G} M(F)
$$

Proof: The family of the univalent and regular functions in both $G_{1}$ and $G$ meet $F\left(z_{0}\right)=0, F^{\prime}\left(z_{0}\right)=1$ are respectively marked as $N\left(G_{1}\right)$ and $N(G), G_{1} \subset G, N(G) \subset N\left(G_{1}\right)$, therefore

$$
\inf _{F \in G_{1}} M(F) \leq \inf _{F \in G} M(F) .
$$

Definition: Suppose $G$ is the convex region of the axial symmetry, the midpoint of the straight line segment of the symmetrical axis in $G$ is named as the midpoint of the symmetrical axis of the convex region.

Property 2: Suppose $G$ is the convex region with axial symmetry which rounded by a piecewise smooth curve $C$. The midpoint of the symmetrical axis is $a$,

$$
R=\max _{\zeta \in C}|a-\zeta|, r(z)=\min _{\zeta \in C}|z-\zeta|, \quad(z \in G),
$$

thus the mapping radius function $R_{G}(z)$ of $G$ meets

$$
r(z) \leq R_{G}(z) \leq R
$$

Proof: Suppose $z \in G, z$ is the center of the circle, $r(z)=\min _{\zeta \in C}|z-\zeta|$ is the radius to the inscribed circular disc $G . G:|z-\xi| \leq r(z), \quad \xi \in C$, thus $G_{1} \subset G . R_{G_{1}}(z) \leq R_{G}(z)$ according to property 1, and $R_{G_{1}}(z)=r(z)$, thus $r(z) \leq R_{G}(z)$.

The circumscribed circular disc to $G G_{2}:|\zeta-a| \leq R$ is made with $a$ as the center of the circle, and the radius as $R=\max _{\zeta \in C}|a-\zeta| . R_{G}(z) \leq R_{G_{2}}(z)$ is obtained according to property 1 . For $R_{G_{2}}(z) \leq R_{G_{2}}(a)=R$, that is, $R_{G}(z) \leq R$. Sum up the above statements, $r(z) \leq R_{G}(z) \leq R$.

Especially, when $r=\min _{\zeta \in c}|a-\zeta|$, the maximum of $R_{G}(z)$ is marked as $R_{G(M)}$, thus,

$$
r \leq R_{G(M)} \leq R
$$

Theorem 1: Suppose $G$ is the convex region rounded by a piecewise smooth curve $C, \zeta \in C$, thus $\lim _{z \rightarrow \zeta} R(z)=0$.

Proof: For formulation (4)

$$
r(\mathrm{z}) \leq R_{G}(\mathrm{z}) \leq R, \frac{1}{R} \leq \frac{1}{R_{G}(\mathrm{z})} \leq \frac{1}{r(\mathrm{z})},
$$

that is the univalent and regular function $f(z)$ that will be changed $G$ into the unit circle $:|w|<1$, and meets $f\left(z_{0}\right)=0, f^{\prime}\left(z_{0}\right)>0$. For each $f(z)$ conform to $\frac{1}{R} \leq f^{\prime}\left(z_{0}\right) \leq \frac{1}{r\left(z_{0}\right)}$, therefore,

that is ,

$$
R_{G}\left(z_{0}\right)=\frac{1-\left|f\left(z_{0}\right)\right|^{2}}{\left|f^{\prime}\left(z_{0}\right)\right|^{2}} \leq R^{2}\left(1-\left|f\left(z_{0}\right)\right|^{2}\right),
$$

$$
R_{G}(z)=\frac{1-|f(z)|^{2}}{\left|f^{\prime}(z)\right|^{2}} \leq R^{2}\left(1-|f(z)|^{2}\right) \quad(z \in G) .
$$

When $\zeta \in C, \lim _{z \rightarrow \zeta}|f(z)|=1$, thus, $\lim _{z \rightarrow \zeta} R(z)=0$.

Theorem 2: If $G$ is the convex region rounded by a piecewise smooth curve $C$, the mapping radius function $R(z)$ of $G$ must reach the maximum in the region $G$.

Proof: Suppose $C$ is the boundary of $G$, the function $R^{*}(z)=\left\{\begin{array}{cc}R(z) & z \in G \\ 0 & z \in C\end{array}\right.$

is continuous in the closed region $\bar{G}$ formed by $G$ and its boundary $C \cdot R^{*}(z)$ has the maximum, but the value of $R^{*}(z)$ on the boundary is zero. The maximum of $R^{*}(z)$ is at some point $a$ in the region $G$, that is the maximum of $R(z)$ is at the point $a$. 
The following three conclusions [2] are explained in order to give another theorem:

If $f(z)$ is the univalent and regular function in the region $G$, then $f^{\prime}(z) \neq 0$;

If $f(z)$ is the regular function in the region $G, f^{\prime}(z)$ is also the regular function in the region $G$;

If $G$ is the convex region with the symmetrical axis rounded by a piecewise smooth curve $C$, and $z=a$ is the midpoint of the symmetrical axis , $|z-a|=r$, make

$$
M(r)=\max _{|z-a|=r, z \in G}|f(z)|,
$$

$M(r)$ is the monotone increasing function about $r$ according to the principal of the maximum modulus of the regular function.

Theorem 3: If $G$ is the convex region with the symmetrical axis rounded by a piecewise smooth curve $C$, the mapping radius function $R_{G}(z)$ of $G$ gains the maximum at the midpoint of the symmetrical axis of $G$.

Proof: Suppose $f(z)$ is the univalent and regular function

which $G$ will transformed into the $|w|<1$ on plane $W$, and meet $f\left(z_{0}\right)=0, f^{\prime}\left(z_{0}\right)>0$, thus, $f^{\prime}(z)$ is still the regular function in $G$. Mark $a$ as the midpoint of the symmetrical axis of $G$, for $M(r)=\max _{|z-a|=r, z \in G}|f(z)|$ is the increasing function of $|z-a|=r$, thus, $f(z)$ and $f^{\prime}(z)$ reach the minimum at the point $a$. Therefore, the mapping radius function in $G \quad R_{G}(z)=\frac{1-|f(z)|^{2}}{\left|f^{\prime}(z)\right|^{2}}$ reaches the maximum at the point $a$.

Deduction 1: If $G$ is the convex region with the symmetrical center rounded by a piecewise smooth curve $C$, the mapping radius function $R_{G}(z)$ of $G$ obtains the maximum at the symmetrical center of $G$.

Deduction 2: If $G$ is the convex region with the symmetrical center rounded by a piecewise smooth curve $C$, the point of the mapping radius function $R_{G}(z)$ of $G$ gains the maximum is in the center of $G$.

\section{References}

[1] Ge Luxin, “Geometric Theories of Functions of a Complex Variable.” [M], Beijing, Science Press. pp.22 27, 1955.

[2] Zhong Yuquan, “Geometric Theories of Functions of a Complex Variable.” [M], Beijing, Higher Education Press. pp.182, 2004. 